\title{
Survival and late toxicities following concurrent chemo-radiotherapy for locally advanced stage III non-small cell lung cancer: findings of a 10-year Australian single centre experience with long term clinical follow up
}

\author{
M. Nazim Abbas ${ }^{1}$, Adeola Ayoola ${ }^{1}$, Sunita Padman ${ }^{1}$, Rajiv Kumar ${ }^{1}$, John Leung ${ }^{2}$, Shahid Ullah ${ }^{3}$, \\ Bogda Koczwara $^{1,4}$, Shawgi Sukumaran ${ }^{1,4}$, Ganessan Kichenadasse ${ }^{1,4}$, Amitesh Roy ${ }^{1,4}$, Alison M. Richards ${ }^{1}$, \\ Jeffrey J. Bowden ${ }^{5}$, Christos S. Karapetis ${ }^{1,4}$ \\ ${ }^{1}$ Department of Medical Oncology, Flinders Medical Centre, Adelaide, SA, Australia; ${ }^{2}$ Genesis Care, Flinders Private Hospital, Adelaide, SA, \\ Australia; ${ }^{3}$ South Australian Health and Medical Research Institute, Adelaide, SA, Australia; ${ }^{4}$ Flinders Centre for Innovation in Cancer, Flinders \\ University, Adelaide, SA, Australia; ${ }^{5}$ Department of Respiratory and Sleep Services, Flinders Medical Centre, Adelaide, SA, Australia \\ Contributions: (I) Conception and design: CS Karapetis, JJ Bowden, A Roy, J Leung, R Kumar; (II) Administrative support: CS Karapetis, AM \\ Richards; (III) Provision of study materials or patients: J Leung, B Koczwara, S Sukumaran, G Kichenadasse, A Roy, JJ Bowden, CS Karapetis; \\ (IV) Collection and assembly of data: MN Abbas, A Ayoola, S Padman, R Kumar, J Leung, AM Richards, CS Karapetis; (V) Data analysis and \\ interpretation: All authors; (VI) Manuscript writing: All authors; (VII) Final approval of manuscript: All authors. \\ Correspondence to: M. Nazim Abbas. Flinders Centre for Innovation in Cancer, Bedford Park, Adelaide, SA 5042, Australia. \\ Email: nazim.abbas@sa.gov.au; drnazimabbas@gmail.com.
}

Background: The preferred management of patients with unresectable locally advanced non-small cell lung cancer (LA-NSCLC) is concurrent chemo-radiotherapy (CRT). Acute CRT-related toxicities are well defined, however, less is known about late toxicities. The aim of the study was to examine the outcomes and late toxicities in Stage III NSCLC treated with CRT.

Methods: A retrospective review of the data from patients with stage III NSCLC treated with CRT was performed between May 2000 and June 2010. Demographics, tumour and treatment characteristics, toxicities and survival data were examined from hospital records of the patients. Progression free survival (PFS) and overall survival (OS) were evaluated by standard Kaplan-Meier survival curves. The censor date was set on 31 October 2016.

Results: Sixty-three patients were identified with a median age of 66.6 years [interquartile range (IQR) 57.2-72.1], two-third $(\mathrm{n}=41,65.1 \%)$ were male, majority were current or ex-smokers $(\mathrm{n}=52,82.5 \%)$, $42(66.7 \%)$ patients had stage IIIB disease and 21 (33.3\%) had stage IIIA disease. The most common histologic subtype was adenocarcinoma 30 (47.6\%). The median PFS and OS of the whole population was 10.6 months (95\% CI, 4.1-17.3 months) and 21 months (95\% CI, 12.7-29.3 months) respectively. The 5-year OS rates for stage IIIA and IIIB were $24 \%$ and $16 \%$ respectively. The 1-, 3- and 5-year OS rates for all patients were $63.5 \%, 46 \%$ and $18.7 \%$ respectively. Acute grade 3 and 4 toxicities included 28 haematological and 17 non-haematological events. The incidence of late toxicities was $58.9 \%$. Thirty-three events of late grade 3 and 4 toxicities were recorded. The most common late toxicity was symptomatic radiation-induced pulmonary fibrosis (39.3\%), others include ototoxicity (7.1\%), persistent dysphagia (7.1\%) and one case of acute myeloid leukaemia. All patients that were alive at the censor date had developed radiation-induced fibrosis with associated symptoms of respiratory insufficiency.

Conclusions: The 5-year OS of patients with stage III NSCLC treated with CRT was in keeping with survival figures reported from prospective clinical trials. There is, however, significant morbidity associated with long-term survival and this should be taken into account when making informed treatment decisions. 


\begin{abstract}
Keywords: Non-small cell lung cancer (NSCLC); chemo-radiotherapy (CRT); long-term adverse effects; late toxicity
\end{abstract}

Submitted Feb 07, 2019. Accepted for publication Sep 03, 2019.

doi: $10.21037 /$ jtd.2019.09.56

View this article at: http://dx.doi.org/10.21037/jtd.2019.09.56

\section{Introduction}

Non-small cell lung cancer (NSCLC) is the fourth most common cancer diagnosed in Australia, and the leading cause of cancer related death in both males and females (1). About $35 \%$ of patients present with unresectable locally advanced NSCLC (LA-NSCLC) (2) and treatment options include radiotherapy alone; sequential (3-6) or concurrent chemo-radiotherapy (CRT) $(7,8)$.

Platinum-based chemotherapy has been extensively studied in combination with radiation therapy for localised unresectable NSCLC. Older regimens include MVP (mitomycin C, vindesine and cisplatin) (9-11), cisplatin/ vinblastine $(12,13)$, and cisplatin/etoposide (14), newer agents include cisplatin/taxanes (15), cisplatin/gemcitabine $(16,17)$, and platinum/irinotecan (18).

There is limited data on late toxicities and clinical outcomes of survivors in patients with stage III NSCLC treated with CRT. The meta-analysis of CRT in LANSCLC confirmed the lack of data on late toxicity(8).

This retrospective study was aimed to explore the clinical outcomes of LA-NSCLC treated with CRT in our institution over a 10-year period with particular focus on late toxicities.

\section{Methods}

Patients diagnosed between May 2000 and June 2010, with stage III NSCLC, treated with CRT at Flinders Medical Centre and Flinders Private Hospital, South Australia, were included in this retrospective analysis. Patients were identified using the relevant International Classification of Diseases (ICD-9) codes (C34.0, C34.1, C34.2, C34.3, C34.8, C34.9) from inpatient and outpatient encounters through the Department of Medical Oncology and South Australia Central Cancer Registry. Data was collected on demographic information, treatment received, acute and late toxicity (graded according to common terminology criteria for adverse events version 3.0) (19), (radiological response according to RECIST criteria) $(20,21)$, date of diagnosis, and date of death. Staging was defined according to the TNM classification system ( $7^{\text {th }}$ version) (22). Late toxicities were defined as those first developed more than 6 months after the last chemotherapy and or radiation treatment dose. This analysis was taken as a quality assurance project and did not require ethics approval from institutional ethics committee.

\section{Statistical analysis}

Data were analysed using STATA version 13.0 (Stata Corp. 2013, College Station, Texas, USA). Means and standard deviation (SD) were calculated for continuous data. Proportions were presented as percentages of the respective denominator (n). Median and interquartile range (IQR) were also calculated for skewed data, progression free survival (PFS) for each patient was calculated from the start of therapy to first evidence of cancer progression. Patients who died before radiological confirmation of disease progression were recorded as having a date of progression as their date of death. Overall survival (OS) for each patient was calculated from the date of the start of treatment to the date of death as recorded in the national death registry or date of last follow up. Both PFS and OS were evaluated by standard Kaplan-Meier survival curves.

The data was censored on 31 October 2016. The incidence rates of acute and late toxicities were calculated.

\section{Results}

Sixty-three patients with stage III NSCLC were identified who received CRT between May 2000 and June 2010. The median age for the cohort was 66.6 years, (IQR 57.2-72.1) and majority of the patients were males $(65.1 \%)$. The majority of patients were current or ex-smokers $(n=52$; $82.5 \%)$. Twenty-one $(33.3 \%)$ patients had stage IIIA, while $42(66.7 \%)$ had stage IIIB NSCLC. Thirty (47.6\%) patients had adenocarcinoma, followed by large cell carcinoma $18(28.6 \%)$, squamous cell carcinoma $12(19 \%)$, and other histology 3 (4.8\%). The patient demographics are summarized in Table 1. 
Table 1 Patient characteristics $(\mathrm{N}=63)$

\begin{tabular}{|c|c|}
\hline Patient characteristics & $\mathrm{N}(\%)$ \\
\hline Male & $41(65.1)$ \\
\hline Female & $22(34.9)$ \\
\hline Age, median (IQR) & $66.6(57.2-72.1)$ \\
\hline \multicolumn{2}{|l|}{ Smoking status } \\
\hline Never & $7(11.1)$ \\
\hline Ex-smoker & $37(58.7)$ \\
\hline Current smoker & $15(23.8)$ \\
\hline Unknown & $4(6.3)$ \\
\hline \multicolumn{2}{|l|}{ Histology } \\
\hline Adenocarcinoma & $30(47.6)$ \\
\hline Large cell & $18(28.6)$ \\
\hline Squamous & $12(19.0)$ \\
\hline Other & $3(4.8)$ \\
\hline \multicolumn{2}{|l|}{ Stage } \\
\hline IIIA & $21(33.3)$ \\
\hline IIIB & $42(66.7)$ \\
\hline \multicolumn{2}{|l|}{ Chemotherapy regimen } \\
\hline Cisplatin/etoposide & $40(63.5)$ \\
\hline Cisplatin/vinblastine & $12(19.0)$ \\
\hline Carboplatin/paclitaxel & $4(6.3)$ \\
\hline Cisplatin/vinorelbine & $3(4.8)$ \\
\hline Docetaxel & $3(4.8)$ \\
\hline Docetaxel/celecoxib & $1(1.6)$ \\
\hline
\end{tabular}

IQR, interquartile range. (75th percentile-25th percentile).

During the first 5-year period, from 2000 to 2005, 20 patients were treated with concurrent CRT and from 2006 to 2010, 43 patients were treated with concurrent CRT. Chemotherapy regimens varied considerably through the decade, with the majority of patients receiving platinumbased doublet chemotherapy (93.6\%). The most commonly used regimen was cisplatin/etoposide in $40(63.5 \%)$ patients, followed by cisplatin/vinblastine in $12(19 \%)$ patients. Docetaxel as monotherapy was used in $3(4.8 \%)$ patients between 2003 and 2006. One patient was treated in a clinical trial with the addition of celecoxib to docetaxel. These regimens are summarized in Table 1.

The radiation dose received by $61(93.8 \%)$ patients was 60 Gray (Gy) in 30 fractions five days per week. A three-

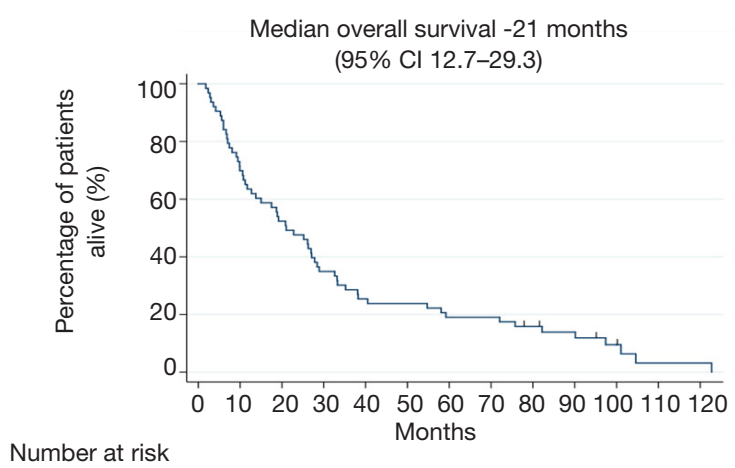

Figure 1 Median overall survival in months for overall population.

Table 2 Median progression free and overall survival by stage (months)

\begin{tabular}{lccccc}
\hline \multirow{2}{*}{ Stage } & \multicolumn{2}{c}{ Progression free survival } & & \multicolumn{2}{c}{ Overall survival } \\
\cline { 2 - 3 } \cline { 5 - 6 } & Month & $95 \% \mathrm{Cl}$ & & Month & $95 \% \mathrm{Cl}$ \\
\hline III & 10.6 & $4.1-17.3$ & & 21 & $12.7-29.3$ \\
IIIA & 11 & $2.6-19.3$ & & 27 & $13.6-40.3$ \\
IIIB & 8 & $0-16.9$ & & 19.2 & $7.4-30.9$ \\
\hline
\end{tabular}

field technique was most commonly used with a V20 (amount of normal lung receiving 20 Gy or more) under $30 \%$ and mean lung doses under 18 Gy achieved in the majority of patients. A V20 of up to $33 \%$ was accepted in a very small number of patients. Spinal cord dose was allowed up to a maximum of $46 \mathrm{~Gy}$. Elective nodal irradiation was abandoned in 2009 and treatment of gross disease with a 1 to $1.5 \mathrm{~cm}$ margin to clinical target volume and a further $1 \mathrm{~cm}$ margin to planning target volume. Image matching was done with weekly portal films. Two patients did not complete their full radiotherapy dose due to CRT-related toxicity. Two patients were treated with lower doses (50 Gy in 25 fractions) due to underlying co-morbidities.

The median follow-up period was 26 months. The minimum follow-up period from date of entry into the series of the last surviving patient was 6 years. Median OS for all stages was 21 months (95\% CI, 12.7-29.3 months) (see Figure 1). Patients with stage IIIA and stage IIIB disease had a median OS of 27 months (95\% CI, 13.6-40.3 months) and 19.2 months (95\% CI, 7.4-30.9 months), respectively (see Table 2). The estimated 1-, 3- and 5-year survival rate for all stages were $63.5 \%, 46 \%$ and $18.7 \%$ respectively. The estimated 5 -year survival rate for stages IIIA and IIIB 


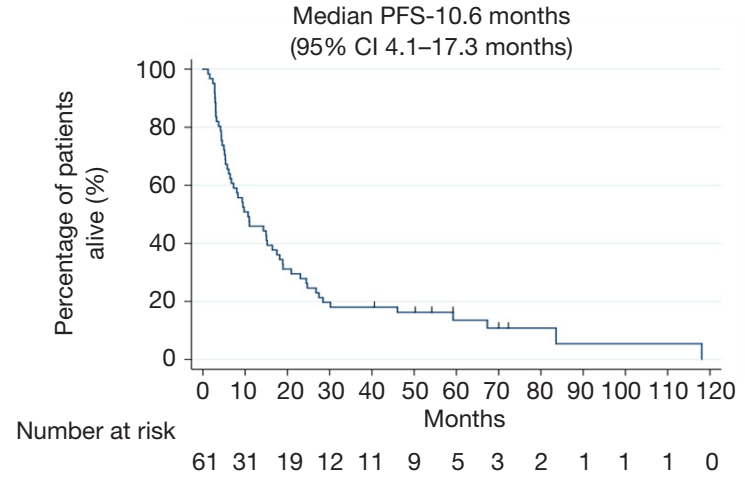

Figure 2 Median PFS in months for overall population. PFS, progression free survival.

Table 3 Frequency of acute toxicities*

\begin{tabular}{lc}
\hline Grade & Events (incidence rate) \\
\hline Haematological & $30(47.6)$ \\
3 & $11(17.5)$ \\
4 & $19(30.2)$ \\
Non-haematological & $21(33.3)$ \\
3 & $20(31.7)$ \\
4 & $1(1.6)$ \\
Total events & 51 \\
\hline
\end{tabular}

*, any toxicity (Grade 3 or 4 ) occurring during or $<6$ months from date of last treatment with chemo-radiation.

disease was $24 \%$ and $16 \%$, respectively.

For all stages, median PFS was 10.6 months (95\% CI, 4.1-17.3 months) (Figure 2). The median PFS for stages IIIA and IIIB was 11 months (95\% CI, 2.6-19.3 months) and 8 months (95\% CI, 0-16.9 months) respectively (see Table 2).

The incidence of acute grade 3 and 4 toxicities was $33.3 \%$ non-hematological and $47.6 \%$ hematological (Table 3). The most common acute grade 4 toxicities were neutropenia (19\%), febrile neutropenia (6.3\%), and oral mucositis $(1.6 \%)$. There was one treatment-related death from a radiation-induced broncho-aortic fistula resulting in acute hemorrhage. One patient was diagnosed with stage III colon cancer within 6 months of completing CRT and subsequently died of metastatic colon cancer. One patient died from metastatic breast cancer; the diagnosis of early breast cancer preceded the NSCLC.

The incidence of late toxicities in 56 recipients alive at
Table 4 Incidence rate of late toxicities

\begin{tabular}{lc}
\hline Late toxicities $^{*}$ & No of events $(\%)$ \\
\hline Cough $^{*}$ & $1(1.8)$ \\
Dysphagia & $4(7.1)$ \\
Neuropathy & $1(1.8)$ \\
Ototoxicity & $4(7.1)$ \\
Radiation fibrosis & $22(39.3)$ \\
Secondary cancer & $1(1.8)$ \\
Total no & $33(58.9)$ \\
\hline
\end{tabular}

*, late toxicity: any toxicity that developed $>6$ months after the date of last treatment with chemotherapy and or radiation; \#, patient developed acute myeloid leukaemia; ${ }^{*}$, cough related to radiation induced pulmonary fibrosis.

6 months was $58.9 \%$ (51.8\% in stage IIIB as compared to $7.1 \%$ in stage IIIA). The results are summarized in Table 4. Specific toxicity grades were not available for these events; identification was dependent on the treating physician's documentation. Common delayed toxicities included radiation fibrosis $(39.3 \%)$, ototoxicity $(7.1 \%)$ and persistent dysphagia (7.1\%).

The event rate (death) on census date was $93.7 \%$ (59 patients). Thirty-three patients (52.4\%) died of NSCLC progression and $1.6 \%$ (1 patient) died of a second cancer.

Four patients $(6.3 \%)$ were alive as at $31^{\text {st }}$ October 2016 and all of them had radiographic features of ongoing late radiation pneumonitis including pleural effusion. Only 2 patients (out of 4) had persistent symptoms however these symptoms did not require any treatment. In these patients, tumour recurrence was excluded by use of serial CT imaging, diagnostic pleural aspiration and PET scans. One patient was diagnosed with concurrent idiopathic pulmonary fibrosis, and had treatment with dexamethasone plus domiciliary oxygen.

Symptomatic pericardial effusion (grade 3 ) due to disease progression was recorded in 1 patient and required pericardiocentesis. Atrial fibrillation (grade 2) was noted in 2 patients and one patient developed congestive cardiac failure (grade 2) in the setting of obstructive sleep apnoea and chronic obstructive pulmonary disease. One patient developed idiopathic dilated cardiomyopathy (grade 3) approximately 11 years after finishing CRT. One patient developed ST segment elevation myocardial infarction in the setting of baseline cardiac risk factors 3 years after completing CRT. Overall in all these patients attribution of cardiac events to CRT was difficult to determine in 
the presence of cardiac risk factors and other potential etiologies.

Less frequent toxicities included one case of peripheral neuropathy; a case of secondary malignancy, acute myeloid leukemia (AML), in a patient treated with cisplatin/ etoposide, who had received a cumulative etoposide dose of $500 \mathrm{mg} / \mathrm{m}^{2}$. The leukemia was diagnosed two years after completion of concurrent CRT and cytogenetic studies revealed $\mathrm{t}(8 ; 21)(\mathrm{q} 22 ; \mathrm{q} 22)$ translocation.

\section{Discussion}

This retrospective review of patients with stage III NSCLC treated with CRT revealed a high incidence $(58.9 \%)$ of late toxicity. There is limited data on late toxicity after CRT in unresectable NSCLC patients. Horinouchi et al. (23) reported grade 1-5 late pulmonary toxicity in $36 \%$ of patients following completion of concurrent cisplatin/vinorelbine chemotherapy and radiation therapy (majority received $60 \mathrm{~Gy}$ in 30 fractions). This included a $4 \%$ incidence of death due to pulmonary toxicity. We report a $39.3 \%$ incidence of radiation fibrosis; this is similar to the outcomes reported by Horinouchi et al. (23). However Rakovitch et al. (24) and Rowell et al. (25) reported no significant increase in the risk of acute pneumonitis in their published data respectively. One factor that may have contributed to the late pulmonary toxicity was the use of two-dimensional radiation therapy in our centre from 2000-2006. Three-dimensional conformal radiation therapy was used from 2007 onwards. A second potential factor was the application of elective nodal radiation from 2000 to 2008, resulting in larger radiation fields. This was considered standard practice during that treatment period. Radiation techniques have improved since the year 2000. The use of intensity modulated radiation therapy, routine use of positron emission tomography for planning and four-dimensional CT are expected to reduce radiation fibrosis significantly. In our series, 2 patients developed chronic pleural effusion, with no evidence of recurrence of malignancy on cytological assessment of pleural fluid and they remained free of cancer recurrence with long-term follow-up.

Radiation esophagitis is a frequent acute complication of CRT in NSCLC but there is limited data on late symptoms of radiation esophagitis. Our data show a low rate of late radiation induced dysphagia of $7.1 \%$ (Table 4). These patients reported persistent dysphagia beyond 6 months after completion of CRT. This is similar to reports in published literature $(24,25)$. The shift from sequential to concurrent chemotherapy has seen a substantial increase in the rate of acute grade 3 to 4 esophagitis, from $4 \%$ to $18 \%$ (8). A review of 4 randomized controlled trials in CRT for LA-NSCLC found at least grade 1 radiation esophagitis in $95 \%$ of patients. Grade 3 or higher grades were seen in $19 \%$ of patients in the first month of treatment, and $33 \%$ by the third month, and $2 \%$ beyond 3 months (26). The difference in toxicity rates could be due to limitations of retrospective data collection, which is not a sensitive tool for grade 1 and 2 toxicities, but better at grade 3 or greater. As noted previously, radiation therapy techniques have improved since the period of treatment of this study, and more modern techniques reduce the dose of radiation delivered to the oesophagus, minimising toxicities to this area.

The platinum-based chemotherapy agent cisplatin causes irreversible sensorineural hearing loss in 20 $40 \%$ of patients (27). We identified $4(7.1 \%)$ cases of ototoxicity, although audiometry was not routinely performed. Ototoxicity has been shown to occur in 33\% of patients following a single administration of cisplatin at $50 \mathrm{mg} / \mathrm{m}^{2}$, with manifestations including hearing loss, tinnitus, otalgia and vestibular disorders (28-30). Rademaker-Lakhai et al. identified patient factors predictive of ototoxicity, which included pre-existing hearing loss, age and renal impairment. Treatment factors predictive of otoxicity were dose $>60 \mathrm{mg} / \mathrm{m}^{2}$, and 2-weekly versus weekly administration. A poor correlation between clinical grading of ototoxicity and audiometry-based assessment was noted (31). To manage the risk of ototoxicity, consideration should be given to monitoring audiometry through treatment, with a switch to lower dose weekly scheduling in the event of early high incidence hearing loss.

Hematological malignancy, particularly AML, is a well described consequence of exposure to cytotoxic therapy, especially with etoposide, a topoisomerase II inhibitor, where risk is related to the cumulative dose administered. In those exposed to topoisomerase II inhibitors, the incidence of AML is between $2 \%$ and $12 \%$, with a mean latency of 2 years. We report an incidence of $1.8 \%$ of AML in our study cohort. The most common chromosomal translocation in chemotherapy associated AML is $t(11: 23)$, however $t(3 ; 21)$, inv (16), $t(8 ; 16), t(15 ; 17), t(9 ; 22)$ and $t(8 ; 21)$ have been seen less frequently $(32)$. In our series, a patient treated with cisplatin/etoposide and RT developed AML with translocation $t(8 ; 21)(q 22 ; q 22) 24$ months after treatment. Given the infrequent rate of secondary 
malignancies, there is little epidemiological data about these complications in patients treated with CRT. Ratain et al. (33) described 4 cases of AML at 13, 19, 28 and 35 months after treatment with etoposide-based combination chemotherapy in patients with NSCLC. Although rare, AML may be a serious complication of etoposide-based CRT.

The absolute survival benefit of concurrent CRT in LA-NSCLC is well known in the literature. The 2 and 5 years absolute survival benefit of $4 \%$ and $2.2 \%$ compared to sequential CRT was reported in the meta-analysis by Aupérin et al. (8). The estimated 5 year survival rate of $18.7 \%$ in our patient population is similar to the published retrospective data from New South Wales (Australia) by Spina et al. (34) who reported a $17 \%$ estimated 5 year survival rate.

Long-term survival is achievable and is a principal treatment goal in the management of stage III NSCLC. The majority of patients, however, do not survive long term and further research is needed to improve patient outcomes. The optimal treatment strategy for inoperable stage III NSCLC now incorporates immune therapy as a consolidation treatment for 1 year after the completion of CRT (35). Our series predates the use of immune therapy. Nevertheless, it is reassuring to see long-term survival approximates that observed from prospective randomised clinical trials of CRT alone. We anticipate further survival benefit will be achieved through the addition of immune therapy in current day routine clinical practice.

Incidence of radiotherapy related cardiac adverse events have been reported to be approximately $33 \%$ in patients with NSCLC (36). Due to the retrospective nature of the study, documentation of cardiotoxicity related to CRT was limited. Six cardiac events were recorded in the study population and none of these were directly attributed to the previous chemoradiotherapy.

This study reports on the late toxicities following CRT in stage III NSCLC, an often under reported outcome in clinical trials. The major limitations in this study include retrospective nature of the data analysed, incomplete documentation of toxicity data, a small sample size, and limited data on cardiotoxicity outcomes following CRT. There is need to establish more robust databases on longterm outcomes of cancer survivors. 'Real world experience' data can capture long-term clinical outcomes, acute and late effects on organs at risk (lung, heart, spinal cord, and oesophagus) and treatment details following disease progression. There is also a need to measure quality of life effects, especially following multi-modality approach.

\section{Conclusions}

Long term survival is an achievable goal when treating inoperable stage III NSCLC with CRT. A high proportion of long-term survivors, however, experience late toxicities, particularly respiratory insufficiency secondary to radiation fibrosis. These late toxicities could impact on quality of life and need to be factored into clinical decision-making.

\section{Acknowledgments}

The co-authors wish to acknowledge the contribution of late Dr. Tony Woo, to the research. He was affiliated with the Adelaide Radiotherapy Centre, Flinders Private Hospital, Bedford Park, Australia.

\section{Footnote}

Conflicts of Interest: The authors have no conflicts of interest to declare.

Ethical Statement: The authors are accountable for all aspects of the work in ensuring that questions related to the accuracy or integrity of any part of the work are appropriately investigated and resolved. This analysis was taken as a quality assurance project and did not require ethics approval from institutional ethics committee.

\section{References}

1. Cancer in Australia: an overview, 2012. Available online: https://www.aihw.gov.au/getmedia/efaa639f-d6b8-4e198d38-bcba7646360e/14757.pdf. Accessed: August 27,2019.

2. Jemal A, Bray F, Center MM, et al. Global cancer statistics. CA Cancer J Clin 2011;61:69-90.

3. Dillman RO, Seagren SL, Propert KJ, et al. A randomized trial of induction chemotherapy plus high-dose radiation versus radiation alone in stage III non-small-cell lung cancer. N Engl J Med 1990;323:940-5.

4. Rolland E, Le Chevalier T, Auperin A, et al. Sequential radio-chemotherapy (RT-CT) versus radiotherapy alone (RT) and concomitant RT-CT versus RT alone in locally advanced non-small cell lung cancer (NSCLC): Two meta-analyses using individual patient data (IPD) from randomised clinical trials (RCTs): A1-04. J Thorac Oncol 2007;2:S309-10.

5. Sause W, Kolesar P, Taylor S IV, et al. Final results of phase III trial in regionally advanced unresectable non- 
small cell lung cancer: Radiation Therapy Oncology Group, Eastern Cooperative Oncology Group, and Southwest Oncology Group. Chest 2000;117:358-64.

6. Sause WT, Scott C, Taylor S, et al. Radiation Therapy Oncology Group (RTOG) 88-08 and Eastern Cooperative Oncology Group (ECOG) 4588: preliminary results of a phase III trial in regionally advanced, unresectable non-small-cell lung cancer. J Natl Cancer Inst 1995;87:198-205.

7. Okawara G, Mackay JA, Evans WK, et al. Management of unresected stage III non-small cell lung cancer: a systematic review. J Thorac Oncol 2006;1:377-93.

8. Aupérin A, Le Péchoux C, Rolland E, et al. Meta-analysis of concomitant versus sequential radiochemotherapy in locally advanced non-small-cell lung cancer. J Clin Oncol 2010;28:2181-90.

9. Fukuoka M, Masuda N, Furuse K, et al. A randomized trial in inoperable non-small-cell lung cancer: vindesine and cisplatin versus mitomycin, vindesine, and cisplatin versus etoposide and cisplatin alternating with vindesine and mitomycin. J Clin Oncol 1991;9:606-13.

10. Gebbia V, Galetta D, Riccardi F, et al. Vinorelbine plus cisplatin versus cisplatin plus vindesine and mitomycin $\mathrm{C}$ in stage IIIB-IV non-small cell lung carcinoma: a prospective randomized study. Lung Cancer 2002;37:179-87.

11. Gridelli C, Perrone F, Palmeri S, et al. Mitomycin C plus vindesine plus etoposide (MEV) versus mitomycin $\mathrm{C}$ plus vindesine plus cisplatin (MVP) in stage IV non-small-cell lung cancer: A phase III multicentre randomised trial. The "Gruppo Oncologico Centro-Sud-Isole' (G.O.C.S.I.). Ann Oncol 1996;7:821-6.

12. Kris MG, Gralla RJ, Kalman LA, et al. Randomized trial comparing vindesine plus cisplatin with vinblastine plus cisplatin in patients with non-small cell lung cancer, with an analysis of methods of response assessment. Cancer Treat Rep 1985;69:387-95.

13. Mylonakis N, Tsavaris N, Bacoyiannis C, et al. A randomized prospective study of cisplatin and vinblastine versus cisplatin, vinblastine and mitomycin in advanced non-small cell lung cancer. Ann Oncol 1992;3:127-30.

14. Albain KS, Crowley JJ, Turrisi AT 3rd, et al. Concurrent cisplatin, etoposide, and chest radiotherapy in pathologic stage IIIB non-small-cell lung cancer: a Southwest Oncology Group phase II study, SWOG 9019. J Clin Oncol 2002;20:3454-60.

15. Segawa Y, Kiura K, Takigawa N, et al. Phase III trial comparing docetaxel and cisplatin combination chemotherapy with mitomycin, vindesine, and cisplatin combination chemotherapy with concurrent thoracic radiotherapy in locally advanced non-small-cell lung cancer: OLCSG 0007. J Clin Oncol 2010;28:3299-306.

16. Byrne MJ, Phillips M, Powell A, et al. Cisplatin and gemcitabine induction chemotherapy followed by concurrent chemoradiotherapy or surgery for locally advanced non-small cell lung cancer. Intern Med J 2005;35:336-42.

17. Vokes EE, Herndon JE 2nd, Crawford J, et al. Randomized phase II study of cisplatin with gemcitabine or paclitaxel or vinorelbine as induction chemotherapy followed by concomitant chemoradiotherapy for stage IIIB non-smallcell lung cancer: cancer and leukemia group B study 9431. J Clin Oncol 2002;20:4191-8.

18. Yamamoto N, Nakagawa K, Nishimura Y, et al. Phase III study comparing second- and third-generation regimens with concurrent thoracic radiotherapy in patients with unresectable stage III non-small-cell lung cancer: West Japan Thoracic Oncology Group WJTOG0105. J Clin Oncol 2010;28:3739-45.

19. National Cancer Institute, Common Terminology Criteria for Adverse Events v3.0 Available online: https://ctep. cancer.gov/protocolDevelopment/electronic_applications/ docs/ctcaev3.pdf. Accessed: August 27, 2019.

20. Therasse P, Arbuck SG, Eisenhauer EA, et al. New guidelines to evaluate the response to treatment in solid tumors. European Organization for Research and Treatment of Cancer, National Cancer Institute of the United States, National Cancer Institute of Canada. J Natl Cancer Inst 2000;92:205-16.

21. Eisenhauer EA, Therasse P, Bogaerts J, et al. New response evaluation criteria in solid tumours: revised RECIST guideline (version 1.1). Eur J Cancer 2009;45:228-47.

22. Goldstraw P, Crowley J, Chansky K, et al. The IASLC Lung Cancer Staging Project: proposals for the revision of the TNM stage groupings in the forthcoming (seventh) edition of the TNM Classification of malignant tumours. J Thorac Oncol 2007;2:706-14.

23. Horinouchi H, Sekine I, Sumi M, et al. Long-term results of concurrent chemoradiotherapy using cisplatin and vinorelbine for stage III non-small-cell lung cancer. Cancer Sci 2013;104:93-7.

24. Rakovitch E, Tsao M, Ung Y, et al. Comparison of the efficacy and acute toxicity of weekly versus daily chemoradiotherapy for non-small-cell lung cancer: a metaanalysis. Int J Radiat Oncol Biol Phys 2004;58:196-203.

25. Rowell NP, O'rourke NP. Concurrent chemoradiotherapy 
in non-small cell lung cancer. Cochrane Database Syst Rev 2004:CD002140.

26. Werner-Wasik M, Paulus R, Curran WJ Jr, et al. Acute esophagitis and late lung toxicity in concurrent chemoradiotherapy trials in patients with locally advanced non-small-cell lung cancer: analysis of the radiation therapy oncology group (RTOG) database. Clin Lung Cancer 2011;12:245-51.

27. Bokemeyer C, Berger CC, Hartmann JT, et al. Analysis of risk factors for cisplatin-induced ototoxicity in patients with testicular cancer. Br J Cancer 1998;77:1355-62.

28. Reddel RR, Kefford RF, Grant JM, et al. Ototoxicity in patients receiving cisplatin: importance of dose and method of drug administration. Cancer Treat Rep 1982;66:19-23.

29. Schaefer SD, Wright CG, Post JD, et al. Cis-platinum vestibular toxicity. Cancer 1981;47:857-9.

30. Strauss M, Towfighi J, Lord S, et al. Cis-platinum ototoxicity: clinical experience and temporal bone

Cite this article as: Abbas MN, Ayoola A, Padman S, Kumar R, Leung J, Ullah S, Koczwara B, Sukumaran S, Kichenadasse G, Roy A, Richards AM, Bowden JJ, Karapetis CS. Survival and late toxicities following concurrent chemo-radiotherapy for locally advanced stage III non-small cell lung cancer: findings of a 10-year Australian single centre experience with long term clinical follow up. J Thorac Dis 2019;11(10):4241-4248. doi: 10.21037/jtd.2019.09.56 histopathology. Laryngoscope 1983;93:1554-9.

31. Rademaker-Lakhai JM, Crul M, Zuur L, et al. Relationship between cisplatin administration and the development of ototoxicity. J Clin Oncol 2006;24:918-24.

32. Felix CA. Secondary leukemias induced by topoisomerasetargeted drugs. Biochim Biophys Acta 1998;1400:233-55.

33. Ratain MJ, Kaminer LS, Bitran JD, et al. Acute nonlymphocytic leukemia following etoposide and cisplatin combination chemotherapy for advanced non-small-cell carcinoma of the lung. Blood 1987;70:1412-7.

34. Spina R, Chu SY, Chatfield M, et al. Outcomes of chemoradiation for patients with locally advanced nonsmall-cell lung cancer. Intern Med J 2013;43:790-7.

35. Antonia SJ, Villegas A, Daniel D, et al. Durvalumab after Chemoradiotherapy in Stage III Non-Small-Cell Lung Cancer. N Engl J Med 2017;377:1919-29.

36. Ming X, Feng Y, Yang C, et al. Radiation-induced heart disease in lung cancer radiotherapy: A dosimetric update. Medicine (Baltimore) 2016;95:e5051. 